Pathophysiology

of Haemostasis and Thrombosis
Pathophysiol Haemost Thromb 2007-08;36:271-274

DOI: $\underline{10.1159 / 000252824}$
Received: September 29, 2008

Accepted after revision: April 27, 2009

\title{
Incidence of Deep Venous Thrombosis in Patients with Erysipelas of the Leg: Prospective Study of 30 Cases in an Emergency Department
}

\author{
Imen Zaghdoudi ${ }^{\mathrm{a}}$ Monia Rezgui ${ }^{\mathrm{a}}$ Wathak Zouaoui ${ }^{\mathrm{b}}$ Takoua Marhbene $^{\mathrm{a}}$ \\ Ali Jendoubi $^{\mathrm{a}}$ Rim El Fatimi ${ }^{\mathrm{a}}$ Nébiha Borsali-Falfoul ${ }^{\mathrm{c}}$ \\ Mohamed Habib Daghfous ${ }^{b}$ Zouhair Jerbi ${ }^{a}$ \\ aEmergency Department and ${ }^{\mathrm{b}}$ Radiology Department, Habib Thameur Hospital, and 'Emergency Department, \\ La Rabta Hospital, Tunis, Tunisia
}

\section{Key Words}

Lower limb erysipelas - Anticoagulation - Deep venous thrombosis

\begin{abstract}
The treatment of lower limb erysipelas is based on antistreptococcal antibiotherapy. The indication of adjuvant anticoagulant therapy is not clear because of the lack of data about the incidence of deep venous thrombosis (DVT) in these patients. We performed a prospective study using a colour Doppler vein exploration combined with ultrasonography within the first $48 \mathrm{~h}$, with evaluation of clinical probability of DVT according to the Wells score to assess the incidence of DVT in patients with erysipelas of the lower limb. Of 30 patients studied, 3 DVT were diagnosed: 2 distal DVT (posterior tibial vein) and 1 proximal DVT (superficial femoral vein) in patients having a clinical score $\geq 3$. Pulmonary embolism was diagnosed in 2 cases. The incidence of DVT in our study was $10 \%$. DVT should be considered in patients with erysipelas of the lower limb having a high pretest clinical probability.

Copyright $\odot 2009$ S. Karger AG, Basel
\end{abstract}

\section{Introduction}

Erysipelas is a non-necrotizing dermo-hypodermatitis mainly caused by Streptococcus pyogenes. It occurs in $10-100$ cases per 100,000 people per year [1]. This incidence is increasing despite the control of streptococcal infections [2]: 2 French studies [3, 4] described a progressive increase in the yearly number of hospitalizations for erysipelas between 1978 and 1991 and between 1959 and 1995. A recent study [5] showed that the yearly age-standardized incidence of erysipelas increased from 1.88 per 1,000 patients in the years $1994-1995$ to 2.49 in $2003-$ 2004 in primary care in Flanders and the northern part of Belgium. The diagnosis of erysipelas is made clinically in the presence of an acute inflammatory plaque characterized by 2 features: the lesions are raised above the level of the surrounding skin, and there is a clear line of demarcation between involved and uninvolved tissue [6]. Other local signs such as lymphangitis or adenopathy and general signs like fever and hyperleucocytosis can be associated. Bacteriologic examinations are not helpful because of the low sensitivity of blood culture and stan-

\section{KARGER}

Fax +4161306 1234

E-Mail karger@karger.ch

www.karger.com
(C) 2009 S. Karger AG, Basel

$1424-8832 / 08 / 0365-0271 \$ 24.50 / 0$

Accessible online at:

www.karger.com/pht
Dr. Imen Zaghdoudi

Emergency Departement, Habib Thameur Hospital

$N^{\circ} 8$, Ali Ben Ayed Street

1008 Montfleury, Tunis (Tunisia)

Tel. +216 98633 528, Tel./Fax +216 71491 227, E-Mail imen.zaghdoudi@rns.tn 
Table 1. Wells score

\begin{tabular}{llcc}
\hline & High & Moderate & Low \\
\hline DVT+ & 3 & 0 & 0 \\
DVT- & 3 & 10 & 14 \\
\hline
\end{tabular}

dard examinations (needle aspiration, skin biopsy) and the delayed positivity of serology [7]. Historically, erysipelas was described in the face $[8,9]$. Actually, erysipelas is localized in lower limbs in more than $90 \%$ of cases bringing about an intrinsic risk of deep venous thrombosis (DVT) of the lower limbs [10]. The treatment is based on anti-streptococcal antibiotherapy [7]. The indication of adjuvant anticoagulant therapy and its modalities are not clearly established.

We conducted a prospective study in the settings of our emergency department to assess the incidence of DVT in patients with lower limbs erysipelas.

\section{Patients}

\section{Inclusion Criteria}

In our study, we enrolled adult patients presenting to the emergency department of the Habib Thameur Hospital (teaching hospital, Tunis, Tunisia) with the diagnosis of erysipelas during 1 year $(01 / 11 / 2006$ to $31 / 10 / 2007)$. The diagnosis is made on the basis of an acute onset $<24 \mathrm{~h}$ and a well-demarcated inflammatory placard (oedematous, red, fiery and tender) uni- or bilaterally localized in the lower limbs. These clinical signs might be associated with tender adenopathy and/or lymphangitis or with general signs like fever $>38^{\circ} \mathrm{C}$ and/or chills. We excluded patients with a diagnosis of necrotizing fasciitis or with previous anticoagulant treatment.

\section{Methods}

For all patients, demographic data (age, sex and antecedents), physical and local examination of the lower limb were noted. A systematic colour Doppler vein exploration combined with ultrasonography (DVCUS) was performed within the first $48 \mathrm{~h}$ by the same operator (ultrasound device ATL $5000 \mathrm{HDI}$ ). The diagnosis of a recent DVT was made in the presence of segmental dilatation of the vein with an increased venous calibre, incompressibility of the vein totally or partially, and the echogenicity of the thrombus.

For each patient, the clinical pretest probability of DVT (low, moderate, high) was assessed according to the Wells score [11]. In-hospital treatment decision was made in the following cases: age $>60$ years, comorbidities (diabetes or cardiovascular disease), systemic toxicity, or severity of local signs.

For out-door patients, penicillin $\mathrm{G}$ is prescribed by intramuscular route, and in the case of allergy, a synergistine (Pyostacine ${ }^{\circledR}$ ) is given orally. For in-house patients, penicillin $\mathrm{G}$ is prescribed by intravenous route (oral Pyostacine in the case of allergy), and a prophylactic anticoagulation by low-molecular-weight heparin is also administered. In case of a confirmation of DVT, low-molecular-weight heparin with a curative dose is given in combination with vitamin $\mathrm{K}$ antagonist (VKA): acenocoumarol (Sintrom ${ }^{\circledR}$ ).

\section{Results}

Thirty patients were enrolled (12 males/18 females), with a mean age of $61.7 \pm 15.3$ years. Nineteen patients were hospitalized and 11 treated as ambulatory patients. Three DVT cases, homolateral to erysipelas, were diagnosed among hospitalized patients ( 2 males/ 1 female), affecting the posterior tibial vein in 2 patients and extending from the inferior third of the superficial femoral vein to the popliteal vein in 1 patient. The Wells score [11] was high in 6 patients, moderate in 10 patients and low in 14 patients (table 1).

The mean age and inflammation markers (C-reactive protein, D-dimers and leucocytes) did not differ significantly in hospitalized patients with or without DVT.

Two asymptomatic bilateral subsegmental pulmonary embolisms (PEs) were diagnosed among the 3 patients with DVT: CT arteriography was normal, but the ventilation-perfusion scanning revealed bilateral subsegmental defects of perfusion with normal ventilation.

The patient without PE underwent oral anticoagulation during 3 months with VKA, then stopped after a DVCUS control showing a total permeability of the posterior tibial vein. For the 2 other patients, VKA was continued during 8 and 12 months, respectively, and then stopped after the ventilation-perfusion scanning control showed improvement.

\section{Discussion}

Erysipelas brings about a favourable environment to thrombosis because of the initial bedridden state, disturbance of coagulation with superficial micro-thrombosis and local fibrin deposition mentioned by Hammar et al. [12] and also because of the underlying disease (congestive heart failure, venous insufficiency). In an open study, these authors showed that fibrinolysis is decreased; fibrinogen and activities of several plasma serine proteinases involved in coagulation were increased during the initial course of erysipelas and even at follow-up.

The incidence of DVT in patients with lower limb erysipelas in our study was $10 \%$. There are few studies where 
Table 2. Incidence of DVT in patients with lower limb erysipelas

\begin{tabular}{lccccl}
\hline Reference & Year & Patients & DVT & DVT, \% & Exploration method \\
\hline Lindblad et al. [14] & 1988 & 43 & 3 & $6.9(1.5-19.1)$ & 125I-fibrinogen uptake test \pm phlebography \\
Mahe et al. [13] & 1992 & 40 & 6 & $15(5.7-29.8)$ & DVCUS \\
Jeune [15] & 1991 & 10 & 0 & $0(0.0-30.9)$ & phlebography \\
Perrot et al. [16] & 2001 & 155 & 4 & $2.6(0.7-6.5)$ & DVCUS \\
Our study & 2008 & 30 & 3 & $10(2.1-26.5)$ & DVCUS \\
\hline
\end{tabular}

Figures in parentheses are $95 \%$ confidence intervals. Ponderated incidence with $95 \%$ confidence interval: $5.8 \%(3.3-9.2)$.

Table 3. Sensitivity and specificity stratified by US technique [19]

\begin{tabular}{lllll}
\hline & \multicolumn{2}{l}{ Sensitivity, \% } & & \multirow{2}{*}{ Specificity, \% } \\
\cline { 2 - 4 } & all DVT & proximal DVT & distal DVT & \\
\hline Compression only & $90.3(88.4-92.0)$ & $93.8(92.0-95.3)$ & $56.8(49.9-66.4)$ & $97.8(97.0-98.4)$ \\
Colour Doppler & $81.7(77.4-85.5)$ & $95.8(85.7-99.5)$ & $43.5(23.2-66.5)$ & $92.7(89.7-95.1)$ \\
Compression plus colour Doppler & $91.1(89.0-93.0)$ & $96.4(94.4-97.9)$ & $75.2(67.7-81.6)$ & $94.3(92.5-95.8)$ \\
\cline { 1 - 2 } & & & &
\end{tabular}

Figures in parentheses are $95 \%$ confidence intervals.

DVT was systematically looked for (table 2): Mahe et al. [13], in a series of 40 patients admitted to the internal medicine department with the diagnosis of erysipelas and explored by systematic DVCUS within the first $48 \mathrm{~h}$, found 6 DVT homolateral to erysipelas, which is an incidence of $15 \%$. Lindblad et al. [14] performed a ${ }^{125}$ I-fibrinogen uptake test for the diagnosis of DVT in 43 patients with erysipelas of the leg. The test was positive in $16 \mathrm{pa}-$ tients, and only 11 patients had confirmation by phlebography. Three DVT cases were diagnosed, but the authors admit the low specificity of the ${ }^{125} \mathrm{I}$-fibrinogen uptake test for the diagnosis of DVT in the setting of erysipelas. Jeune [15] explored 10 patients with systematic phlebography, and no DVT was found. Finally, Perrot et al. [16] reported a larger series of 155 patients explored by a systematic DVCUS at admission and at discharge from the hospital: 1 DVT is diagnosed at admission and 3 at discharge, $2 / 3$ contralateral to erysipelas. Two asymptomatic PEs were diagnosed by ventilation-perfusion scanning.

The Wells score [11] allowed the identification of patients at high risk of thromboembolism. Indeed, no DVT was diagnosed in moderate- or low-risk patients.

Plasma dosage of D-dimers (ELISA method) shows positive values in all our patients $(>500 \mathrm{ng} / \mathrm{ml})$ regardless of the presence of DVT, acting rather as a marker of the inflammation and the infection process. Mazzolai et al. [17] also concluded that D-dimer testing is not recommended for the exclusion of DVT in out-patients with lower limb erysipelas.

The confirmation of DVT in our patients (2 distal and 1 proximal DVT) was made by colour Doppler vein exploration with a study of the compressibility with ultrasounds (US). In fact, the diagnostic accuracy of US for DVT varies according to the technique used [18]. In the United States, Canada and the Netherlands, the examination is limited to proximal veins and often limited to US compression only. In France, Italy and Spain, the exploration is complete and includes distal and proximal veins, and US compression is often associated with colour Doppler exploration. A recent meta-analysis [19] identified 100 cohorts comparing US with venography in patients with suspected DVT and established its diagnostic performance with regard of the technique used and the localization of DVT (table 3).

The specificity of the technique employed for our patients is satisfactory; however, the sensitivity, especially in the sub-popliteal veins, is less optimal. This fact could lead us to under-estimate the incidence of DVT in our patients, but clinical follow-up during hospitalization and after discharge did not confirm this hypothesis. 


\section{Conclusion}

The incidence of DVT in patients with lower limb erysipelas is $10 \%$ in our study. The combination of clinical evaluation of DVT probability and the exploration by
DVCUS refines the diagnosis and the management. As a matter of fact, DVT should be considered in patients with erysipelas of the lower limb having a high pretest clinical probability, but larger studies are needed to confirm this result.

\section{References}

1 Erysipelas and necrotizing fasciitis: management. Consensus conference. French Society of Dermatology. Ann Med Interne 2000;151: 465-470.

$>2$ Efstratiou A: Group A streptococci in the 1990s. J Antimicrob Chemother 2000;45:312.

$\checkmark 3$ Chartier C, Grosshans E: Erysipelas: an update. Int J Dermatol 1996;35:779-781.

-4 Bernard P, Bedane C, Mounier M, Denis F, Bonnetblanc J: Bacterial dermohypodermal infections: incidence and actual place for a streptococcal origin. Ann Dermatol Venereol 1995;122:495-500.

5 Bartholomeeusen S, Vandenbroucke J, Truyers C, Buntinx F: Epidemiology and comorbidity of erysipelas in primary care. Dermatology 2007;215:118-122.

6 Vaillant L: Diagnostic criteria for erysipelas. Ann Dermatol Venereol 2001;128:326-333.

$\checkmark 7$ Stevens DL, Bisno AL, Chambers HF, Everett ED, Dellinger P, Goldstein EJC, Gorbach SL, Hirschmann JV, Kaplan EL, Montoya JG, Wade JC: Practice guidelines for the diagnosis and management of skin and soft tissue infections. Clin Infect Dis 2005;41:13731406.
8 Bratton RL, Nesse RE: St Anthony's fire: diagnosis and management of erysipelas. Am Fam Phys 1995;51:401-404.

$\checkmark 9$ Bisno AL, Stevens DL: Streptococcal infections of skin and soft tissues. N Engl J Med 1996;334:240-245.

10 Schmit JL: A prospective study on erysipelas and infectious cellulitis: how are they dealt with in hospital? Ann Dermatol Venereol 2001;128:334-337.

11 Wells PS, Anderson DR, Bormanis J, Guy F, Mitchell M, Gray L, Clement C, Robinson KS, Lewandowski B: Value of assessment of pretest probability of deep vein thrombosis in clinical management. Lancet 1997;350 1795-1798.

12 Hammar H, Sverdrup B, Borglund E, Blombäck M: Coagulation and fibrinolytic systems during the course of erysipelas and necrotizing fasciitis and the effect of heparin. Acta Derm Venereol 1985;65:495-503.

13 Mahe A, Destelle JM, Bruet A, Mathé C, Tuot D, Taveau JF, Quevauvilliers J, Fendler JP: Deep venous thrombosis in patients with erysipelas of the leg. Presse Med 1992;21: 1022-1024.
14 Lindblad B, Wallmark E, Berqvist D, Cronberg S: Low specificity of ${ }^{125} \mathrm{I}$-fibrinogen uptake test for the diagnosis of deep vein thrombosis in patients with erysipelas of the leg. Acta Med Scand 1988;224:399-400.

15 Jeune R: Erysipelas: epidemiologic, clinical and therapeutic data. Ann Dermatol Venereol 1991;118:545-546.

16 Perrot JL, Perrot S, Laporte Simitsidis S: Is anticoagulant therapy useful when treating erysipelas? Ann Dermatol Venereol 2001; 128:352-357.

17 Mazzolai L, Haesler E, Milesi I, Hayoz D: $\mathrm{D}$-dimers testing is not recommended for the exclusion of deep vein thrombosis in outpatients with lower limb erysipelas. Thromb Haemost 2002;88:880.

18 Quere I, Galanaud JP, Becker F, Laroche JP, Righini M, Levesque H: Distal deep-venous thrombosis: diagnostic and therapeutic issues. Rev Med Interne 2008;29:491-497.

19 Goodacre S, Sampson F, Thomas S, Van Beek E, Sutton A: Systematic review and metaanalysis of the diagnostic accuracy of ultrasonography for deep vein thrombosis. BMC Med Imaging 2005;5:6. 